\title{
UNLINKING AND UNKNOTTEDNESS OF MONOTONE LAGRANGIAN SUBMANIFOLDS
}

\author{
GEORGIOS DIMITROGLOU RIZELL AND JONATHAN DAVID EVANS
}

\begin{abstract}
Under certain topological assumptions, we show that two monotone Lagrangian submanifolds embedded in the standard symplectic vector space with the same monotonicity constant cannot link one another and that, individually, their smooth knot type is determined entirely by the homotopy theoretic data which classifies the underlying Lagrangian immersion. The topological assumptions are satisfied by a large class of manifolds which are realised as monotone Lagrangians, including tori. After some additional homotopy theoretic calculations, we deduce that all monotone Lagrangian tori in the symplectic vector space of odd complex dimension at least five are smoothly isotopic.
\end{abstract}

\section{Part 1. Introduction}

Consider two $n$-dimensional embedded submanifolds, $L_{1}$ and $L_{2}$, of $2 n$-dimensional Euclidean space $\mathbf{R}^{2 n}$. We say that $L_{1}$ links $L_{2}$ if $L_{1}$ is homologically essential in the complement of $L_{2}$. We say that $L_{1}$ and $L_{2}$ are not linked if each one is nullhomologous in the complement of the other.

When $L_{1}$ and $L_{2}$ are diffeomorphic then we can ask if they are isotopic through embedded submanifolds. If they are not then we say they are relatively knotted. In high dimensions, $n \geq 4$, the issue of knottedness is related to the question of linking via a theorem of Haefliger and Hirsch [26]. We discuss this in more depth in Section 6.2 below.

Now suppose that $\mathbf{R}^{2 n}$ is equipped with its standard symplectic form $\omega_{0}$ and that $L_{1}$ and $L_{2}$ are required to be Lagrangian submanifolds (that is, $\omega_{0}$ vanishes on the tangent spaces of $L_{1}$ and $L_{2}$ ). The question of whether $L_{1}$ and $L_{2}$ can be linked or relatively knotted is subtle and has been much studied, [35, 19, 21, 20, 18, 8, 29, 27, 22]. We review some known results in Section 3 below.

\section{StATEMENT Of Results}

The theorems we prove concern knotting and linking of Lagrangian submanifolds in the standard symplectic vector space $\mathbf{C}^{n}$, or more generally in a subcritical Stein manifold. We need to restrict attention to a certain class 
of Lagrangian embeddings: the monotone ones, see Definition 2.4. Note that by torus we always mean a product of circles.

Theorem A. Let $X$ be a subcritical Stein manifold and let $K_{1} \geq K_{2}>0$ be real numbers. An embedded $K_{2}$-monotone Lagrangian torus cannot link an embedded $K_{1}$-monotone Lagrangian torus. In particular, two embedded $K$-monotone Lagrangian tori are not linked.

This theorem fails if we let $K_{2}>K_{1}$ : consider a pair of concentric circles with different radii in $\mathbf{C}$. The theorem is clear for circles in $\mathbf{C}$ by an area argument. It is less intuitively clear why it should be true in higher dimensions.

Theorem B. Suppose $L_{1}$ and $L_{2}$ are embedded monotone Lagrangian $n$-tori in $\mathbf{C}^{n}, n \geq 4$, and suppose that they are homotopic through Lagrangian immersions. Then they are smoothly isotopic through embeddings (not necessarily Lagrangian embeddings).

This is a consequence of Theorem $\mathrm{A}$ using Haefliger-Hirsch theory. By performing some homotopy computations inspired by [8] we also derive:

Corollary C. If $n \geq 5$ is odd then all monotone Lagrangian $n$-tori in $\mathbf{C}^{n}$ are smoothly isotopic after reparametrisation.

Note that this is really a rigidity theorem for Lagrangians and the proof uses hard tools (pseudoholomorphic curves) in an essential way: if we were to relax the Lagrangian condition to totally real then there are totally real embeddings of tori representing all isotopy classes of smooth embeddings (by the h-principle for totally real embeddings) and the isotopy classes of smooth embeddings are in bijection with $H_{1}(L ; Y(n))$ where

$$
Y(n)=\left\{\begin{array}{lll}
\mathbf{Z} & \text { if } n \equiv 1 & \bmod 2 \\
\mathbf{Z} / 2 & \text { if } n \equiv 0 & \bmod 2
\end{array}\right.
$$

(see Section 6.2). There are many examples of Hamiltonian non-isotopic monotone Lagrangian tori in high-dimensional symplectic vector spaces due to Chekanov and Schlenk [13] which are, reassuringly, known to be smoothly (in fact Lagrangian) isotopic. In Section 8 we will construct some examples of relatively knotted monotone tori with non-homotopic Gauss maps when $n$ is even. These are similar to the smoothly knotted Lagrangian $S^{1} \times S^{3}$ examples found in [8].

Both of these theorems will be proved in greater generality below, see Theorem 5.1 and Theorem 6.1 respectively. In particular, we will not require $L_{1}$ and $L_{2}$ to be tori, but the torus is the simplest manifold satisfying the topological conditions we require. Indeed for Theorem $\mathrm{A}$ we do not even 
require that the two Lagrangians are diffeomorphic. We prove Corollary C in Section 6.5 .

In Section 7]we will prove a harder result (Theorem 7.1):

Theorem D. Suppose $L_{1}$ and $L_{2}$ are embedded Lagrangian copies of $S^{1} \times S^{n-1}$ in $\mathbf{C}^{n}$ and suppose that they are homotopic through Lagrangian immersions. If

- $n=2 k+2>4$ and $L_{1}, L_{2}$ have minimal Maslov number $2 k+2$ or

- $n=2 k+1>4$ and $L_{1}, L_{2}$ are monotone

then they are smoothly isotopic through embeddings (not necessarily Lagrangian embeddings).

This result was proved for $n=4,8$ by Borrelli [8], even without the assumption on the minimal Maslov number, using completely different methods. It is mysterious to us that our technique cannot deal with the case $n=4$, but this restriction is needed to rule out certain bad limiting behaviour of punctured holomorphic curves under neck-stretching. We will also prove:

Corollary E. Let $n$ be an integer, $n \geq 5$. If $n$ is odd then all monotone Lagrangian embeddings of $S^{1} \times S^{n-1}$ in $\mathbf{C}^{n}$ are smoothly isotopic after reparametrisation. If $n$ is even then all Lagrangian embeddings of $S^{1} \times S^{n-1}$ in $\mathbf{C}^{n}$ with minimal Maslov number $n$ are smoothly isotopic after reparametrisation.

\section{BASIC DEFINITIONS}

Let $L$ be an oriented $n$-manifold. Equip $L$ with a Riemannian metric and let $\mathrm{Fr}^{+}(L)$ denote the bundle of positive orthonormal frames on $L$. Let $(X, \omega)$ be a symplectic manifold equipped with a compatible almost complex structure $J$ and corresponding almost Kähler metric $g$. Let $\operatorname{Fr}_{U}(X)$ denote the bundle of unitary frames on $X$.

Definition 2.1. An immersion $f: L \leftrightarrow X$ is called Lagrangian if $f^{*} \omega=0$.

Given a Lagrangian immersion $f$, equip $L$ with the metric $f^{*} g$. One defines a Lagrangian frame map

$$
\operatorname{Fr}(f): \mathrm{Fr}^{+}(L) \rightarrow \operatorname{Fr}_{U}(X)
$$

which sends an orthonormal frame of $T_{p} L$ to the pushed-forward frame considered as a unitary basis of $T_{f(p)} X$. Note that the R-span of a unitary basis of $T_{x} X$ is a Lagrangian $n$-plane and that all Lagrangian $n$-planes arise this way. In particular, $U(n)$ acts transitively on the oriented Lagrangian Grassmannian $\Lambda^{+}(n)$ of Lagrangian $n$-planes and the stabiliser is $S O(n)$, acting in the usual way on orthonormal frames in a fixed Lagrangian $n$-plane; 
so $\Lambda^{+}(n) \cong U(n) / S O(n)$. If we define $\Lambda(X)$ to be the Lagrangian Grassmann bundle of all Lagrangian $n$-planes in tangent spaces of $X$ then we see $\operatorname{Fr}_{U}(X)$ is an $S O(n)$-bundle over $\Lambda(X)$ and the map Fr $f$ defined above is an $S O(n)$-equivariant bundle map living over the Lagrangian Gauss map

$$
\Lambda(f): L \rightarrow \Lambda(X)
$$

which sends $p \in L$ to $f_{*}\left(T_{p} L\right) \in \Lambda(X)_{f(p)}$.

Theorem 2.2 (Gromov [25], Lees [33, Theorem 1 and Corollary 2.2]). Two Lagrangian immersions

$$
f_{1}, f_{2}: L \leftrightarrow X
$$

are homotopic through Lagrangian immersions if and only if the maps

$$
\operatorname{Fr}(f)_{1}, \operatorname{Fr}(f)_{2}: \operatorname{Fr}^{+}(L) \rightarrow \operatorname{Fr}_{U}(X)
$$

are homotopic as $S O(n)$-equivariant bundle maps. Moreover, given an $S O(n)$ equivariant bundle map $\Phi: \operatorname{Fr}^{+}(L) \rightarrow \operatorname{Fr}_{U}(X)$ such that the underlying map $\phi: L \rightarrow X$ satisfies

$$
\left[\phi^{*} \omega\right]=0 \in H^{2}(L ; \mathbf{R})
$$

there exists a Lagrangian immersion $F: L \leftrightarrow X$ homotopic to $\phi$ with $\operatorname{Fr}(F)$ homotopic to $\Phi$.

Mostly we consider Lagrangian submanifolds in the standard symplectic vector space $\mathbf{C}^{n}$. The tangent bundle of $\mathbf{C}^{n}$ is canonically trivialised by translation of vectors to the origin. We write

$$
P_{x}: T_{x} \mathbf{C}^{n} \rightarrow T_{0} \mathbf{C}^{n}
$$

for this translation map. In particular we may identify $(S O(n)$-equivariantly) the Lagrangian Grassmann bundle $\Lambda\left(\mathbf{C}^{n}\right)$ with the product

$$
\Lambda\left(\mathbf{C}^{n}\right) \cong \Lambda^{+}(n) \times \mathbf{C}^{n} .
$$

In this trivialisation we consider the Lagrangian Gauss and frame maps as maps

$$
\operatorname{Fr}(f): \operatorname{Fr}^{+}(L) \rightarrow U(n), \quad \Lambda(f): L \rightarrow \Lambda^{+}(n) .
$$

Note that an $S O(n)$-equivariant bundle map $\mathrm{Fr}^{+}(L) \rightarrow U(n)$ is a section of the associated $U(n)$-bundle, which is the complexified frame bundle $\operatorname{Fr}_{\mathbf{C}}(L)$, a principal $U(n)$-bundle. This means that $L$ admits a Lagrangian immersion in $\mathbf{C}^{n}$ if and only if its complexified tangent bundle is trivial and that two Lagrangian frame maps are $S O(n)$-equivariantly homotopic if and only if the corresponding trivialisations of the complexified tangent bundle are homotopic. The difference between two trivialisations of the complexified tangent bundle comprises a map $L \rightarrow U(n)$ and hence $S O(n)$ equivariant homotopy classes of Lagrangian frame map correspond (noncanonically) one-to-one with homotopy classes of maps $L \rightarrow U(n)$.

It is well-known that $H^{1}\left(\Lambda^{+}(n) ; \mathbf{Z}\right) \cong \mathbf{Z}$ and that this cohomology group is generated by the Maslov class $\mu$ [3]. 
Definition 2.4. Let $\lambda_{0}$ be the standard Liouville form

$$
\lambda_{0}=\sum_{k=1}^{n} x_{k} d y_{k}
$$

on $\mathbf{C}^{n}$ and $\omega_{0}=d \lambda_{0}$ the standard symplectic 2-form. Let $f: L \leftrightarrow \mathbf{C}^{n}$ be a Lagrangian immersion. The symplectic area class of $f$ is the cohomology class

$$
a(f):=\left[f^{*} \lambda_{0}\right] \in H^{1}(L ; \mathbf{R}) .
$$

The Maslov class of $f$ is the cohomology class

$$
\mu(f):=f^{*} \mu \in H^{1}(L ; \mathbf{R}) .
$$

We say that $f$ is $K$-monotone if

$$
a(f)=K \mu(f)
$$

for some $K>0$.

More generally if $(X, \omega)$ is a symplectic manifold and $f: L \rightarrow X$ a Lagrangian submanifold, there is an area homomorphism

$$
a(f): H_{2}(X, L ; \mathbf{Z}) \rightarrow \mathbf{R}
$$

obtained by integrating $\omega$ over relative chains.

Definition 2.5. Define the infimal disc area of a Lagrangian embedding $f: L \rightarrow$ $X$ to be

$$
A(f)=\inf \left\{a(f)(\beta) \mid \beta \in H_{2}(X, L ; \mathbf{Z}), a(f)(\beta)>0\right\} .
$$

Monotone Lagrangians provide a particularly convenient setting for doing Lagrangian Floer theory [37,6]. This is because the area of a disc controls its Maslov index which controls the expected dimension of the moduli space and hence bubbling phenomena which reduce area also reduce expected dimension, so the boundary of a moduli space will have smaller expected dimension than the moduli space itself. There are good reasons to study monotone Lagrangians in their own right.

Theorem 2.7 ([23, Theorem D]). In the Gromov-Lees h-principle one can require that all Lagrangian immersions are monotone.

Restrictions on embeddings of monotone Lagrangians (as opposed to immersions) are therefore truly rigidity theorems.

Monotone Lagrangians in $\mathbf{C}^{n}$ arise naturally in the Lagrangian mean curvature flow as self-similarly contracting solutions [24]. They also exhibit 
special properties that are not shared by a general Lagrangian submanifold. For instance, orientable monotone Lagrangians in $\mathbf{C}^{3}$ are all products $S^{1} \times \Sigma_{g}$ [23. Theorem B]; counterexamples to the nonmonotone version of this statement can be constructed by applying Polterovich connectsum to remove double points of Lagrangian immersions obtained by an h-principle.

\section{CONTEXT}

The current techniques for understanding knottedness or linking of Lagrangian submanifolds fall into several categories:

3.1. Luttinger surgery. The papers [35, 19, 8] use Luttinger surgery on a hypothetical Lagrangian submanifold with specified self-linking or knotting properties to produce an impossible symplectic manifold. These have the drawback that one must study Lagrangians diffeomorphic to $S^{1} \times S^{k}$, $k=1,3,7$, for which one can define Luttinger surgery.

Theorem 3.1 (Luttinger [35]). There exist isotopy classes of embedded tori in $\mathbf{C}^{2}$ which do not contain Lagrangian embeddings.

Theorem 3.2 (Luttinger [35], Eliashberg-Polterovich [19], Borrelli [8]). Suppose $k \in\{1,3,7\}$. If $S^{1} \times S^{k} \cong L \subset \mathbf{C}^{1+k}$ is a Lagrangian submanifold, $W: D_{\rho, g}^{*} L \rightarrow \mathbf{C}^{1+k}$ is a Weinstein neighbourhood, $\sigma: L \rightarrow S^{1}$ is the projection to the first factor and $L^{\prime}$ is the image under $W$ of the graph of the 1-form $\rho^{\prime} d \sigma$ for some $\rho^{\prime}<\rho$ then $L^{\prime}$ does not link $L$.

In the language of Section 6.2 below, this theorem identifies the HaefligerHirsch field of the Lagrangian embedding and this is enough to determine the smooth knot type when the dimension is sufficiently large. This argument, due to Borrelli, is reproduced in Section 6.4 below. Using it, Borrelli observes:

Corollary 3.3 (Borrelli [8]). Suppose $k \in\{3,7\}$. The smooth isotopy class of a Lagrangian submanifold $S^{1} \times S^{k} \subset \mathbf{C}^{k+1}$ is determined by the homotopy class of the Lagrangian Gauss map. In other words, two Lagrangian $S^{1} \times S^{k} \subset \mathbf{C}^{k+1}$ with homotopic Lagrangian Gauss maps are not relatively knotted.

3.2. Perturbing the symplectic form. The papers [18, 29] perturb the symplectic structure to make a two-dimensional Lagrangian into a symplectic submanifold, choose an almost complex structure making this submanifold into a holomorphic curve and then perturb the almost complex structure to perform isotopies of the holomorphic curve. These have the disadvantage that one must work in very special four-dimensional situations. 
Theorem 3.4 ([18, Genus 0, 1], [29, Genus $>1])$. If $\Sigma$ is an orientable closed surface and $L \subset T^{*} \Sigma$ is a Lagrangian submanifold homologous to the zero section then $L$ is smoothly isotopic to the zero-section.

3.3. Filling with discs. The paper [20] proves a 'local unknottedness theorem' in four dimensions, showing that all Lagrangian planes asymptotic to a linear Lagrangian plane are unknotted.

Theorem 3.5. A Lagrangian embedding $\mathbf{R}^{2} \rightarrow \mathbf{C}^{2}$ which agrees with the embedding of a linear Lagrangian plane $\Pi$ outside a compact set is isotopic (via an ambient compactly supported Hamiltonian isotopy) to П.

The technique of proof is to construct an ambient submanifold of codimension one containing the Lagrangian via the method of filling by holomorphic discs. The submanifold thus constructed is also required to satisfy certain conditions on its characteristic foliation. An isotopy of the Lagrangian with a plane is then constructed explicitly.

3.4. Holomorphic foliations. Hind's papers [27, 28] use symplectic field theory and neck-stretching arguments to put Lagrangian spheres into a special position with respect to a pseudoholomorphic foliation. Neck-stretching arguments were also used in [22, 34] to disjoin a Lagrangian sphere from a fixed collection of symplectic submanifolds and reduce various knotting problems to those studied by Hind or [7] to problems on connectivity of spaces of symplectic ball packings. When these methods work they produce very strong results, but the drawback is that they require foliations by holomorphic curves, and such foliations are only well-behaved in dimension four. They also work best for Lagrangian spheres and it has proved difficult to approach Lagrangian tori this way.

3.5. This paper. Our methods are completely different in character and rely on the existence of many holomorphic discs with boundary on one of the Lagrangians to produce a nullhomology of that Lagrangian. A neckstretching argument is used to ensure that this nullhomology can be made disjoint from the other Lagrangian.

Related work on a Floer theoretic approach to homological inclusion maps for Lagrangian submanifolds is discussed in [1, 2].

\section{Part 2. Proofs}

\section{HOLOMORPHIC DISCS WHICH AVOID A LAGRANGIAN}

If $(X, L)$ is a pair consisting of a symplectic ambient manifold and a Lagrangian submanifold, we denote by $\operatorname{Hur}_{*}(X, L ; \mathbf{Z})$ the image of the Hurewicz 
homomorphism in relative homology. Let $D_{\rho, g}^{*} L$ denote the radius $\rho$ closed disc subbundle of the cotangent bundle $T^{*} L$ for a metric $g$ and $S_{\rho, g}^{*} L$ its boundary.

Theorem 4.1. Let $L_{1}$ and $L_{2}$ be manifolds and suppose that $L_{2}$ admits a metric $g$ with no contractible geodesics. Suppose that

$$
\begin{aligned}
& f_{1}: L_{1} \rightarrow X \\
& f_{2}: L_{2} \rightarrow X
\end{aligned}
$$

are Lagrangian embeddings into a symplectic manifold $(X, \omega)$ (either closed or convex at infinity). Suppose that $A\left(f_{1}\right) \leq A\left(f_{2}\right)$ and that there exists a class $\beta \in \operatorname{Hur}_{2}\left(X, L_{1} ; \mathbf{Z}\right)$ with $a\left(f_{1}\right)(\beta)=A\left(f_{1}\right)$.

Then there exists an almost complex structure on $X$ such that all J-discs with boundary on $L_{1}$ representing $\beta$ are disjoint from $L_{2}$. Moreover, $J$ can be chosen to be regular for the moduli problem of finding discs in the clss $\beta$.

For details of neck-stretching and SFT compactness we refer to the paper [15]. See also [11].

Proof. Let

$$
W: D_{\rho, g}^{*} L_{2} \rightarrow X
$$

be a symplectic embedding, extending $f_{2}$, given by Weinstein's neighbourhood theorem. Let $0<\rho^{\prime}<\rho$ and let $\alpha$ be the contact form on $S_{\rho^{\prime}, g}^{*} L_{2}$ (given by minus the pullback of the Liouville form). The Reeb flow of $\alpha$ is precisely the cogeodesic flow of $g$ and we are assuming there are no contractible closed geodesics. There is an $\epsilon>0$ and a symplectic embedding of

$$
\left((-\epsilon, \epsilon) \times S_{\rho^{\prime}, g}^{*} L_{2}, d\left(e^{r} \alpha\right)\right)
$$

into $X$ as a collar neighbourhood of $W\left(S_{\rho^{\prime}, g}^{*} L_{2}\right)$. Here $r$ denotes the coordinate on the interval $(-\epsilon, \epsilon)$. Let $J_{0}$ be an almost complex structure on $W\left(D_{\rho, g}^{*} L_{2}\right)$ which preserves the contact structure, is compatible with $d\left(e^{r} \alpha\right)$, sends $\partial_{r}$ to the Reeb direction and is $r$-invariant on the collar neighbourhood. Construct a neck-stretching sequence $J_{t}$ as in [15, Section 2.7].

If the theorem is false then for all $t \in[0, \infty)$ there exists a $J_{t}$-holomorphic disc $u_{t}:\left(D^{2}, \partial D^{2}\right) \rightarrow\left(X, L_{1}\right)$ with boundary on $L_{1}$ representing the class $\beta$ such that $u_{t}\left(D^{2}\right) \cap L_{2} \neq \emptyset$. By the SFT compactness theorem [15, Theorem 2.9] we can find a sequence $t_{i} \rightarrow \infty$ such that $u_{i}$ Gromov-Hofer converges (after reparametrisations) to a holomorphic building $u=u_{\bar{W}} \cup u_{S_{1}} \cup \cdots \cup$ $u_{S_{\ell}} \cup u_{\bar{V}}$ where: $\bar{W}$ denotes the symplectic completion of the Weinstein neighbourhood (so $\bar{W} \cong T^{*} L_{2}$ ) and $\bar{V}$ denotes the symplectic completion of $V=X \backslash W\left(D_{\rho, g}^{*}\right)$ (so $\left.\bar{V} \cong X \backslash L_{2}\right) ; u_{\bar{W}}$ denotes the component in $\bar{W}$, $u_{\bar{V}}$ denotes the component in $\bar{V}$ and $u_{S_{k}}$ denote components in the intermediate symplectisation levels. Note that a punctured finite-energy curve 


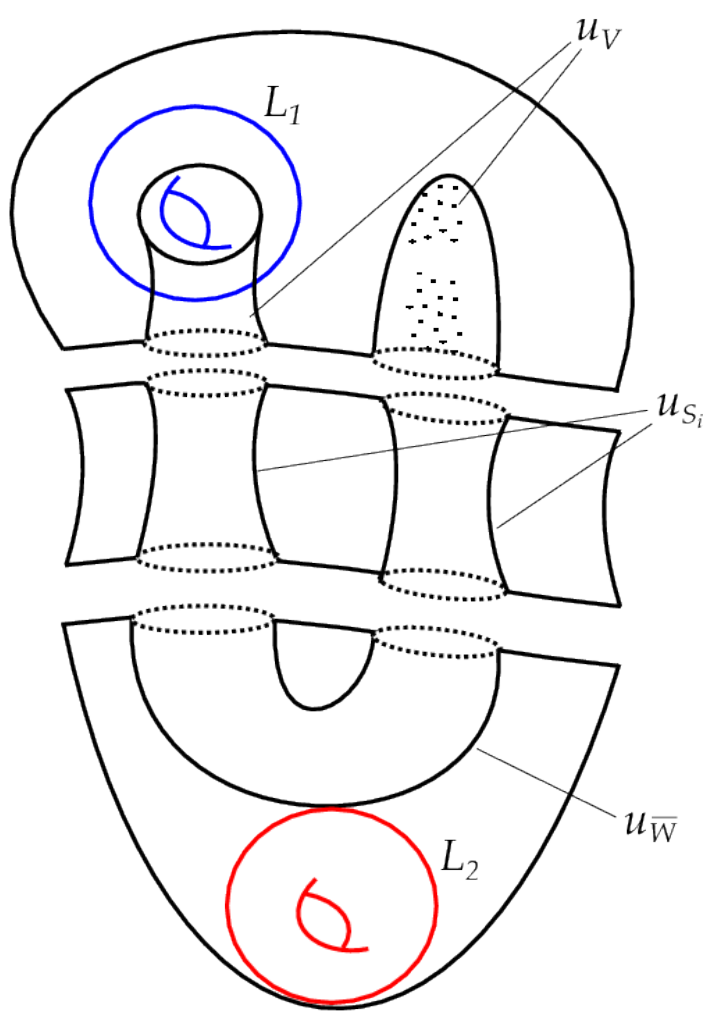

FIGURE 1. The Gromov-Hofer limit must contain a finiteenergy plane as a component of $u_{V}$. This plane (shaded in the figure) has large area since it can be considered as a disc with boundary on $L_{2}$. This contradicts the conservation of area in the limit.

in $\bar{V} \cong X \backslash L_{2}$ is asymptotic to a collection of geodesics in $L_{2}$ and we can compactify it to get a compact topological surface with boundary on $L_{2}$.

Since the domain of $u_{t}$ is a plane there must exist a component $v$ of the building whose domain is a plane. The components of the building are asymptotic to closed Reeb orbits. Since there are no contractible Reeb orbits, $v$ has image in $\bar{V} \cong X \backslash L_{2}$. The asymptotic Reeb orbit of $v$ is a loop in $L_{2}$ and hence we can think of $v$ as a topological disc with boundary on $L_{2}$. Its symplectic area is therefore at least $a_{2}$. Moreover there is another component of $u_{\bar{V}}$ with boundary on $L_{1}$. However, by [15, Definition 2.7(e), Theorem 2.9] the areas of the components of $u_{\bar{V}}$ sum to give $A_{1}(\beta)=a_{1} \leq a_{2}$. Since these summands are all positive [15, Corollary 2.11], we get a contradiction. 
We now show that we can choose $J$ regular for the moduli problem. Since $\beta$ is a minimal homology class of discs we know that pseudoholomorphic discs representing $\beta$ are somewhere injective (by [31, Proposition 4.1]) and hence we can achieve transversality for a Baire set of almost complex structures. Let $J_{k}$ be a sequence of almost complex structures from this Baire set such that $J_{k} \rightarrow J$ as $k \rightarrow \infty$ where $J$ is the almost complex structure we have already constructed. Suppose that for all $k$ is a $J_{k}$-disc $u_{k}$ representing $\beta$ with $u_{k}\left(D^{2}\right) \cap L_{2} \neq \emptyset$. There is a Gromov convergent subsequence $u_{k_{j}}$. Since $\beta$ is minimal there is no bubbling and the limit is a $J$-disc representing $\beta$ intersecting $L_{2}$, which is a contradiction.

\section{UNLINKING OF LAGRANGIANS}

Theorem 5.1. Suppose that $L_{1}$ and $L_{2}$ are n-manifolds such that:

- $L_{2}$ admits a metric with no contractible geodesics,

- $H^{n-1}\left(L_{2} ; \mathbf{Z}\right)$ is torsionfree,

- the cohomology of the universal cover of $L_{1}$ vanishes in odd degrees,

- $L_{1}$ is orientable and spin.

Let $f_{k}: L_{k} \rightarrow \mathbf{C}^{n}, k=1,2$, be Lagrangian embeddings and suppose that

- $L_{1}$ is monotone,

- $L_{1}$ links $L_{2}$.

Then

$$
A\left(f_{2}\right)<A\left(f_{1}\right)
$$

where $A\left(f_{k}\right)$ are defined by Equation (2.6).

Proof. By a theorem of Damian [16] and its sharpening [23, Proposition 7] we know that for a regular $J$ there is a free homotopy class $\beta$ of loops on $L_{1}$ with Maslov index 2 such that, when $J$ is regular, the moduli space

$$
\mathcal{M}_{0,1}(\beta, J)
$$

(of $J$-holomorphic discs with boundary on $L_{1}$ representing $\beta$ and having one boundary marked point) contains a component $M$ such that the evaluation map

$$
\text { ev : } M \rightarrow L_{1}
$$

has nonzero degree, say $d$. Note that the homology class of $\beta$ is minimal by monotonicity. Assume that $J$ is given by Theorem 4.1 so that no discs in $M$ intersect $L_{2}$. Let $N$ denote the moduli space of $J$-holomorphic discs from $M$ with a marked point on the interior. Note that $\partial N=M$. Then $N$ gives a nullhomology of $[M]=d\left[L_{1}\right]$ in $\mathbf{C}^{n} \backslash L_{2}$. Since $H_{n}\left(\mathbf{C}^{n} \backslash L_{2}, \mathbf{Z}\right) \cong$ 
$H^{n-1}\left(L_{2} ; \mathbf{Z}\right)$ is torsionfree by assumption, this implies that $L_{1}$ is nullhomologous in $\mathbf{C}^{n} \backslash L_{2}$.

For monotone Lagrangian tori one does not require the full lifted Floer homology used by Damian or the proposition from [23] to get the existence of these holomorphic discs; one can get away with an argument, due to Buhovsky, using the quantum product structure on Floer cohomology and the Oh spectral sequence: see [12, Theorem 2].

\section{UNKNOTTEDNESS OF LAGRANGIANS}

\subsection{Statement of results.}

Theorem 6.1. Let $n \geq 4$ and let $L$ be an $n$-manifold satisfying the following conditions:

(1) L admits a metric $g$ with no nonconstant contractible geodesics,

(2) the cohomology group $H^{n-1}(L ; \mathbf{Z})$ is torsionfree.

Suppose that $f_{1}: L \rightarrow \mathbf{C}^{n}$ is a monotone Lagrangian embedding and suppose that there exists a submersion $\sigma: L \rightarrow S^{1}$ with connected fibre satisfying

$$
\mu\left(f_{1}\right)=\sigma^{*}\left[\frac{d \theta}{2 \pi}\right] .
$$

Suppose that $f_{2}: L \rightarrow \mathbf{C}^{n}$ is another monotone Lagrangian embedding such that the Lagrangian frame maps are $S O(n)$-equivariantly homotopic when restricted to the complement $L_{x}$ of a point $x \in L$ :

$$
\left.\left.\operatorname{Fr}\left(f_{1}\right)\right|_{L_{x}} \simeq_{S O(n)} \operatorname{Fr}\left(f_{2}\right)\right|_{L_{x}} .
$$

Then $f_{2}$ is smoothly isotopic to $f_{1}$ through embeddings (not necessarily through Lagrangian embeddings).

Note that the frame maps being equivariantly homotopic when restricted to the complement of a point is a weaker condition than being equivariantly homotopic over the whole of $L$. We used the latter condition in the statements of Theorems $B$ and $D$ to make them more readable, but only the weaker assumption is needed.

Corollary 6.2. For $n \geq 4$, the smooth isotopy class of a monotone Lagrangian embedding of the n-torus in $\mathbf{C}^{n}$ is determined by the $S O(n)$-equivariant homotopy type of the Lagrangian frame map.

Proof. The flat $n$-torus, $n \geq 4$, satisfies Conditions 1 and 2 of Theorem 6.1 . If $f_{1}: T^{n} \rightarrow \mathbf{C}^{n}$ is a monotone Lagrangian embedding then by [12] or [16] we know there is a relative homology class $\beta \in H_{2}\left(\mathbf{C}^{n}, T^{n} ; \mathbf{Z}\right) \cong H_{1}(L ; \mathbf{Z})$ with $\mu(\beta)=2$. In particular, half the Maslov class $\frac{1}{2} \mu\left(f_{1}\right) \in H^{1}(L ; \mathbf{Z})$ is 
a primitive cohomology class: if $\mu\left(f_{1}\right)=k \nu$ for some class $\nu \in H^{1}(L ; \mathbf{Z})$ then $1=\frac{1}{2} \mu(\beta)=k \nu(\beta)$ so $k= \pm 1$. Since $L$ is a torus, any primitive homology class $c \in H_{1}(L ; \mathbf{Z})$ can be represented as $\sigma^{*}\left[\frac{d \theta}{2 \pi}\right]$ for a circlevalued function $\sigma$ satisfying the conditions of Theorem 6.1. The corollary is now immediate.

Remark 6.3. By Theorem 2.2. knowing the $S O(n)$-equivariant homotopy type of the Lagrangian frame map is the same as knowing the homotopy class of Lagrangian immersions. Therefore Theorem B is a direct consequence of Corollary 6.2 .

6.2. Haefliger-Hirsch theory. In their paper [26] Haefliger and Hirsch classified smooth embeddings of compact $n$-manifolds into $\mathbf{C}^{n}$ up to isotopy. We explain their results in this section.

Definition 6.4. Let $L$ be a closed, oriented, connected n-manifold and suppose that $f: L \rightarrow \mathbf{C}^{n}$ is an embedding. Let $x \in L$ be a point and define $L_{x}=L \backslash\{x\}$ and $f_{x}=\left.f\right|_{L_{x}}$. A Haefliger-Hirsch field, $v \in \Gamma\left(f_{x}^{*}\left(T \mathbf{C}^{n}\right)\right)$, is a vector field along $L_{x}$ with the following properties:

- $v$ is normal to $L_{x}$,

- if $v$ is extended to a section of $f^{*}\left(T \mathbf{C}^{n}\right)$ by cutting off in a ball around $x$ then the pushoff $L^{\prime}$ of $L$ along this extended vector field is nullhomologous in $\mathbf{C}^{n} \backslash L_{x}$.

Lemma 6.5 (See [26]). Given an embedding $f$ there is a Haefliger-Hirsch field $v$ which is unique up to homotopy.

Define a fibre bundle

$$
\mathcal{V}_{2 n, n+1} \rightarrow L
$$

whose fibre is the Stiefel manifold $V_{2 n, n+1} \cong S O(2 n) / S O(n-1)$ of $n+1$ frames in $\mathbf{R}^{2 n}$. This bundle is associated to the $S O(n)$-frame bundle of $L$ by the action of $S O(n)$ on the first $n$ vectors of the $n+1$-frame. Given an embedding $f$ with Haefliger-Hirsch field $v$ we can define a section $s(f)$ of $\left.\mathcal{V}_{2 n, n+1}\right|_{L_{x}}$. Giving a section of this associated bundle is equivalent to giving an $S O(n)$-equivariant map from the oriented frame bundle $\mathrm{Fr}^{+}\left(L_{x}\right)$ to the Stiefel manifold. We just send an oriented orthonormal frame $F$ in $T_{y} L$ to

$$
s(f)(y)=\left(P_{f(y)} f_{*}(F), P_{f(y)} v\right) \in V_{2 n, n+1} .
$$

where $P$ denotes the trivialisation defined by Equation (2.3). We are writing the $n+1$-frame as $(G, w)$ where $G$ consists of the first $n$ vectors and $w$ is the last. We call the $S O(n)$-equivariant map

$$
s(f): \operatorname{Fr}^{+}\left(L_{x}\right) \rightarrow V_{2 n, n+1}=S O(2 n) / S O(n-1)
$$

the Haefliger-Hirsch map. 
Theorem 6.6 (See [26]). Suppose $n \geq 4$. If $f^{\prime}: L \rightarrow \mathbf{C}^{n}$ is another embedding then it is smoothly isotopic to $f$ if and only if the Haefliger-Hirsch maps $s(f)$ and $s\left(f^{\prime}\right)$ are homotopic as $S O(n)$-equivariant maps $\operatorname{Fr}^{+}\left(L_{x}\right) \rightarrow V_{2 n, n+1}=$ $S O(2 n) / S O(n-1)$.

If $\Gamma\left(\left.\mathcal{V}_{2 n, n+1}\right|_{L_{x}}\right)$ denotes the space of sections of the Stiefel manifold bundle over $L_{x}$ (i.e. the space of Haefliger-Hirsch maps) Haefliger and Hirsch identify

$$
\begin{aligned}
\pi_{0}\left(\Gamma\left(\left.\mathcal{V}_{2 n, n+1}\right|_{L_{x}}\right)\right) & =H^{n-1}\left(L ; \pi_{n-1}\left(V_{2 n, n+1}\right)\right) \\
& =\left\{\begin{array}{lll}
H_{1}(L ; \mathbf{Z}) & \text { if } n \equiv 1 & \bmod 2 \\
H_{1}(L ; \mathbf{Z} / 2) & \text { if } n \equiv 0 & \bmod 2 .
\end{array}\right.
\end{aligned}
$$

This is an application of obstruction theory and of the fact that $V_{2 n, n+1}$ is $n-2$-connected and $\pi_{n-1}\left(V_{2 n, n+1}\right)=\mathbf{Z}$ (respectively $\mathbf{Z} / 2$ ) when $n$ is odd (respectively even); see [39, Theorem 3.16] for this latter computation.

6.3. Identifying the Haefliger-Hirsch field. Take a metric on $L$. The gradient of $\sigma$ is a nowhere-vanishing vector field $\nabla \sigma$. Under the musical isomorphism with the cotangent bundle it is identified with the 1-form $d \sigma$. Let $J$ be a compatible almost complex structure on $T^{*} L$ and consider $\nabla \sigma$ as a vector field defined along the zero-section.

Let $\left\{L_{t}: L \rightarrow T^{*} L\right\}_{t \in(-1,1)}$ be a 1-parameter family of embedded submanifolds such that

- $L_{0}$ is just the inclusion of the zero-section,

- $\left.\frac{d}{d t}\right|_{t=0} L_{t}(y)=J\left(L_{0}\right)_{*} \nabla \sigma(y)$ for all $y \in L$,

- $\operatorname{im}\left(L_{t}\right) \cap \operatorname{im}\left(L_{0}\right)=\emptyset$ for $0 \neq|t|<\epsilon$.

Then for $\epsilon>0, L_{\epsilon}$ is smoothly isotopic in $T^{*} L \backslash L$ to the inclusion of the graph of the 1-form $d \sigma$.

In the setting of Theorem 6.1. let $f_{1}: L \rightarrow \mathbf{C}^{n}$ be a monotone Lagrangian embedding. Let $W: D_{\rho}^{*} L \rightarrow \mathbf{C}^{n}$ be a Weinstein neighbourhood of $f_{1}(L)$ in $\mathbf{C}^{n}$ and $L^{\prime}$ be the image of the graph of $\rho^{\prime} d \sigma$ under $W$ for some $0<\rho^{\prime}<\rho$.

Lemma 6.7. If $L$ is $K$-monotone with $\mu\left(f_{1}\right)=\sigma^{*}\left[\frac{d \theta}{2 \pi}\right]$ the Lagrangian $L^{\prime}$ is $K^{\prime}$-monotone where

$$
K^{\prime}=K-2 \pi \rho^{\prime}<K \text {. }
$$

Proof. Since $L$ and $L^{\prime}$ are Lagrangian isotopic their Lagrangian Gauss maps are homotopic so the Maslov class is unchanged. We need to compute the symplectic area class

$$
\left[\lambda_{0}\right] \in H^{1}\left(L^{\prime} ; \mathbf{R}\right) \cong \operatorname{Hom}\left(H_{1}\left(L^{\prime} ; \mathbf{R}\right), \mathbf{R}\right) .
$$


Let $\alpha$ denote minus the (canonical) Liouville 1 -form on $D_{\rho, g}^{*} L$ so that $d \alpha$ is the canonical symplectic form. Note that $W^{*} \lambda_{0}-\alpha$ is closed because the Weinstein embedding is symplectic, and its cohomology class is $\left[\lambda_{0}\right] \in$ $H^{1}(L ; \mathbf{R})$. Note that

$$
H^{1}\left(D_{\rho, g}^{*} L ; \mathbf{R}\right) \stackrel{i^{*}}{\cong} H^{1}(L ; \mathbf{R})
$$

where $i: L \rightarrow D_{\rho, g}^{*}$ is the inclusion, therefore $W^{*} \lambda_{0}=\alpha+G+d F$ where $G$ is a form representing $\left(i^{*}\right)^{-1}\left[\lambda_{0}\right]$ and $F$ is a function. Let $i_{\rho^{\prime} d \sigma}: L^{\prime} \rightarrow D_{\rho, g}^{*}$ be the inclusion of $L^{\prime}$ into the Weinstein neighbourhood and $f_{1}^{\prime}=W \circ i_{\rho^{\prime} d \sigma}$ the embedding of $L^{\prime}$ into $\mathbf{C}^{n}$. Then

$$
\begin{aligned}
{\left[\left(f_{1}^{\prime}\right)^{*} \lambda_{0}\right] } & =\left[i_{\rho^{\prime} d \sigma}^{*} W^{*} \lambda_{0}\right] \\
& =\left[i_{\rho^{\prime} d \sigma}^{*}(\alpha+G+d F)\right] \\
& =-\rho^{\prime}[d \sigma]+K \mu\left(f_{1}\right)
\end{aligned}
$$

by definition of the canonical 1 -form $-\alpha$ and by $K$-monotonicity. Since $[d \sigma]=2 \pi \mu\left(f_{1}\right)$ by assumption we get monotonicity with constant

$$
K^{\prime}=K-2 \pi \rho^{\prime} .
$$

Lemma 6.8. The vector field $v=J\left(f_{1}\right)_{*} \nabla \sigma$ restricted to the complement of a point $x \in L$ is a Haefliger-Hirsch field for $f_{1}$.

Proof. As we observed above, the pushoff of $L$ along $v$ is smoothly isotopic to a $K^{\prime}$-monotone Lagrangian $L^{\prime}$ where $K^{\prime}<K$. Since $L^{\prime}$ admits a metric without contractible geodesics, we know that $L^{\prime}$ is aspherical by the method of Lyusternik and Fet [36]: otherwise one could apply Birkhoff's minimax technique to a nontrivial higher homotopy class and obtain a contractible geodesic. Therefore $L_{2}=L$ and $L_{1}=L^{\prime}$ satisfy the assumptions of Theorem 5.1. Therefore $L^{\prime}$ is nullhomologous in the complement of $L$; in particular we see that $v$ is a Haefliger-Hirsch field.

6.4. Unknottedness. Theorem 6.1 follows immediately from Lemma 6.8 and the following:

Lemma 6.9. Suppose that $f_{1}, f_{2}: L \rightarrow \mathbf{C}^{n}$ are Lagrangian embeddings such that the Maslov class $\mu$ is represented by $\sigma^{*}\left[\frac{d \theta}{2 \pi}\right]$ for a circle-valued function $\sigma$ with no critical points and such that $J\left(f_{i}\right)_{*} \nabla \sigma$ are the Haefliger-Hirsch fields. If the Lagrangian frame maps associated to $f_{i}$ are homotopic when restricted to $L_{x}$, then the respective Haefliger-Hirsch maps are homotopic.

Proof. Suppose that $\mathcal{F}_{t}, t \in[1,2]$, is a homotopy of $S O(n)$-equivariant Lagrangian frame maps

$$
\mathcal{F}_{t}: \operatorname{Fr}^{+}\left(L_{x}\right) \rightarrow U(n)
$$


with $\mathcal{F}_{i}=\operatorname{Fr}\left(f_{i}\right)$ for $i=1,2$. For each frame $e=\left(e_{1}, \ldots, e_{n}\right) \in \operatorname{Fr}^{+}\left(L_{x}\right)$ define the cofficients $a(e)_{k}$ by $\nabla \sigma=\sum_{k=1}^{n} a(e)_{k} e_{k}$. Now

$$
\begin{gathered}
s\left(\mathcal{F}_{t}\right): \operatorname{Fr}^{+}\left(L_{x}\right) \rightarrow V_{2 n, n+1}=S O(2 n) / S O(n-1), \\
s\left(\mathcal{F}_{t}\right)\left(e_{1}, \ldots, e_{n}\right)=\left(\mathcal{F}_{t} e_{1}, \ldots, \mathcal{F}_{t} e_{n}, J \sum_{k=1}^{n} a(e)_{k} \mathcal{F}_{t}\left(e_{k}\right)\right)
\end{gathered}
$$

is a homotopy of $S O(n)$-equivariant maps connecting the Haefliger-Hirsch maps

$$
\begin{gathered}
s\left(f_{i}\right): \operatorname{Fr}^{+}\left(L_{x}\right) \rightarrow V_{2 n, n+1}=S O(2 n) / S O(n-1), \\
s\left(f_{i}\right)\left(e_{1}, \ldots, e_{n}\right)=\left(\left(f_{i}\right)_{*} e_{1}, \ldots,\left(f_{i}\right)_{*} e_{n}, J\left(f_{i}\right)_{*} \nabla \sigma\right)
\end{gathered}
$$

for $i=1,2$.

We will now prove a more precise result which will allow us to prove Corollary C. Recall from Section 6.2 that given two embeddings $f_{1}, f_{2}: L \rightarrow \mathbf{C}^{n}$, there is a difference class $\epsilon\left(f_{1}, f_{2}\right) \in H^{n-1}\left(L ; \pi_{n-1}\left(V_{2 n, n+1}\right)\right)$ which vanishes if and only if the respective Haefliger-Hirsch maps are homotopic. We begin by showing the following more general statement about this difference class.

Let $U(n) \rightarrow S O(2 n) / S O(n-1)=V_{2 n, n+1}$ be the map induced by the inclusion of $U(n)$ in $S O(2 n)$ and let

$$
u_{n}: \pi_{n-1}(U(n)) \rightarrow \pi_{n-1}\left(V_{2 n, n+1}\right)
$$

be the induced map on $(n-1)$ th homotopy groups.

Suppose that $n$ is even. Let $e_{n / 2} \in H^{n-1}(U(n) ; \mathbf{Z})$ be the characteristic class in $U(n)$ induced by the $\frac{n}{2}$ th Chern class in $B U(n)$ via suspension, that is by pulling back this Chern class in $H^{n}(B U(n), \star ; \mathbf{Z})$ to a relative class $H^{n}(E U(n), U(n) ; \mathbf{Z})$ and mapping it by the inverse of the connecting homomorphism in the long exact sequence of the pair $(E U(n), U(n))$ to $H^{n-1}(U(n) ; \mathbf{Z})$. Fixing a trivialisation of $\mathbf{C} \otimes T L$, the Lagrangian framemap induced by the respective embededings are can be represented by a map $F_{i}: L \rightarrow U(n)$. The difference class

$$
\Delta\left(f_{1}, f_{2}\right):=F_{1}^{*}\left(c_{n / 2}\right)-F_{2}^{*}\left(c_{n / 2}\right) \in H^{n-1}(L, \mathbf{Z})
$$

is well-defined independently of the choice of trivialisation and vanishes if the Lagrangian frame maps $F_{i}$ are homotopic.

Proposition 6.10. Suppose that $f_{1}, f_{2}: L \rightarrow \mathbf{C}^{n}$ are Lagrangian embeddings such that the Maslov class $\mu$ is represented by $\sigma^{*}\left[\frac{d \theta}{2 \pi}\right]$ for a circle-valued function $\sigma$ with no critical points and such that $J\left(f_{i}\right)_{*} \nabla \sigma$ are the Haefliger-Hirsch fields. Then $\epsilon\left(f_{1}, f_{2}\right)$ vanishes when $n$ is odd and

$$
\epsilon\left(f_{1}, f_{2}\right)=\Delta\left(f_{1}, f_{2}\right) \bmod 2
$$


when $n$ is even. Furthermore, if the Lagrangian frame maps are homotopic when restricted to the $(n-2)$-skeleton of $L$, then $\epsilon\left(f_{1}, f_{2}\right)$ vanishes for $n \geq 5$.

We begin by noting two lemmata.

Lemma 6.11. Let $L$ be an oriented manifold with a nowhere-vanishing vector field $v$. Denote by $\mathrm{Fr}^{+}(L)$ the principal bundle of oriented frames and by $\operatorname{Fr}^{\uparrow}(L) \subset$ $\mathrm{Fr}^{+}(L)$ the principal $S O(n-1)$-bundle of frames whose first vector is $v /|v|$. An $S O(n)$-equivariant map $\mathrm{Fr}^{+}(L) \rightarrow V_{2 n, n+1}$ restricts to an $S O(n-1)$-equivariant map $\operatorname{Fr}^{\uparrow}(L) \rightarrow V_{2 n, n+1}$ and an $S O(n-1)$-equivariant map

$$
\alpha: \operatorname{Fr}^{\uparrow}(L) \rightarrow V_{2 n, n+1}
$$

extends uniquely to an $S O(n)$-equivariant map

$$
\hat{\alpha}: \operatorname{Fr}^{+}(L) \rightarrow V_{2 n, n+1} \text {. }
$$

Proof. The map $\hat{\alpha}$ is given by

$$
\hat{\alpha}(e)=\phi \alpha\left(\phi^{-1} e\right)
$$

where $e \in S O(n)=\operatorname{Fr}_{p}^{+}(L)$ is a frame at $p \in L$ and $\phi$ is an element of $S O(n)$ for which $\phi^{-1} e$ has $v /|v|$ as its first vector.

Lemma 6.12. Suppose that $n \geq 5$. Let $p: U(n) \rightarrow S O(2 n) / S O(n-1)=$ $V_{2 n, n+1}$ be the map induced by the inclusion of $U(n)$ in $S O(2 n)$. Then the induced map

$$
u_{n}: \pi_{n-1}(U(n)) \rightarrow \pi_{n-1}\left(V_{2 n, n+1}\right)
$$

vanishes.

Proof. When $n>1$ we have

$$
\pi_{n-1}(U(n))=\left\{\begin{array}{lll}
0 & \text { if } n \equiv 1 & \bmod 2 \\
\mathbf{Z} & \text { if } n \equiv 0 & \bmod 2
\end{array}\right.
$$

by Bott periodicity. So when $n$ is odd, $u_{n}$ vanishes automatically.

The map $u_{n}$ factors as

$$
\pi_{n-1}(U(n)) \rightarrow \pi_{n-1}(S O(2 n)) \stackrel{\Upsilon_{n}}{\longrightarrow} \pi_{n-1}\left(V_{2 n, n+1}\right) .
$$

The map $\Upsilon_{n}: \pi_{n-1}(S O(2 n)) \rightarrow \pi_{n-1}\left(V_{2 n, n+1}\right)$ lives in the long exact sequence

$$
\pi_{n-1}(S O(2 n)) \stackrel{\Upsilon_{n}}{\longrightarrow} \pi_{n-1}\left(V_{2 n, n+1}\right) \rightarrow \pi_{n-2}(S O(n-1)) \rightarrow \pi_{n-2}(S O(2 n))
$$


Assume $n$ is even. Since $\pi_{n-1}\left(V_{2 n, n+1}\right)=\mathbf{Z} / 2$ we see that $\Upsilon_{n}$ vanishes if its cokernel is nontrivial. When $n \equiv 2 \bmod 8$, using the tables on the first and second pages of [30], we get

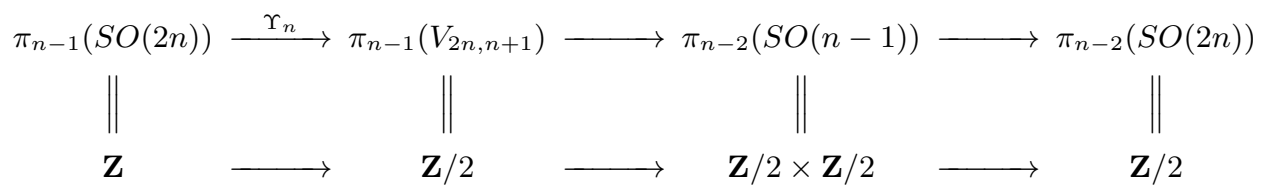

and it is clear that $\Upsilon_{n}$ has nontrivial cokernel. Note that when $n$ is not congruent to 2 modulo 8 , the rightmost term vanishes by Bott periodicity and hence the cokernel of $\Upsilon_{n}$ is precisely the group $\pi_{n-2}(S O(n-1))$. From the tables in [30], we see that $\pi_{n-2}(S O(n-1))$ is always nontrivial unless $n=2,4,8$.

When $n=8, \pi_{7}(S O(16))=\mathbf{Z}$ by Bott periodicity and the map to $\pi_{7}\left(V_{16,9}\right)$ is surjective. However the map $\mathbf{Z}=\pi_{7}(U(8)) \rightarrow \pi_{7}(S O(16))=\mathbf{Z}$ is multiplication by 2 so the composite $\pi_{7}(U(8)) \rightarrow \pi_{7}\left(V_{16,9}\right)$ is trivial, which is what we wanted to show.

To see that the map $\mathbf{Z}=\pi_{7}(U(8)) \rightarrow \pi_{7}(S O(16))=\mathbf{Z}$ is given by multiplication by 2 note that it arises in the exact sequence

$$
\cdots \rightarrow \pi_{7}(U(8)) \rightarrow \pi_{7}(S O(16)) \rightarrow \pi_{7}(S O(16) / U(8)) \rightarrow \pi_{6}(U(8)) \rightarrow \cdots
$$

and that $\pi_{6}(U(8))=0$ by Bott periodicity and $\pi_{7}(S O(16) / U(8))=\mathbf{Z} / 2$.

Proof of Proposition 6.10. Consider the sub-fibre bundle $\operatorname{Fr}^{\uparrow}\left(L_{x}\right) \subset \operatorname{Fr}^{+}\left(L_{x}\right)$ of the frame bundle which consists of frames whose first basis vector points in the direction of $\nabla \sigma$. Observe that this is naturally a principal $S O(n-1)$ bundle.

The Haefliger-Hirsch maps

$$
\begin{gathered}
s\left(f_{i}\right): \operatorname{Fr}^{+}\left(L_{x}\right) \rightarrow V_{2 n, n+1}=S O(2 n) / S O(n-1), \\
s\left(f_{i}\right)\left(e_{1}, \ldots, e_{n}\right)=\left(e_{1}, \ldots, e_{n}, J\left(f_{1}\right)_{*} \nabla \sigma\right)
\end{gathered}
$$

which are $S O(n)$-equivariant maps, restrict to $S O(n-1)$-equivariant maps

$$
s\left(f_{i}\right)^{\uparrow}: \operatorname{Fr}^{\uparrow}\left(L_{x}\right) \rightarrow V_{2 n, n+1} .
$$

Since the first vector is $e_{1}=\nabla \sigma$, the restrictions $s\left(f_{i}\right)^{\uparrow}$ factorise as

$$
s\left(f_{i}\right)^{\uparrow}=p \circ \operatorname{Fr}\left(f_{i}\right)^{\uparrow},
$$

where

$$
\operatorname{Fr}\left(f_{i}\right)^{\uparrow}: \operatorname{Fr}^{\uparrow}\left(L_{x}\right) \rightarrow U(n)
$$

is the restriction of the Lagrangian frame map, and where the projection

$$
p: U(n) \rightarrow S O(2 n) / S O(n-1)=V_{2 n, n+1}
$$

is induced by the inclusion $U(n) \subset S O(2 n)$. 
Furthermore, for a fixed choice of complex trivialisation of $\mathbf{C} \otimes T L$, the Lagrangian frame maps are expressed as maps

$$
F_{i}: L \rightarrow U(n) .
$$

Furthermore, using the $S O(n-1)$-equivariant map $F: \operatorname{Fr}^{\uparrow}(L) \rightarrow U(n)$ which identifies a given frame with a matrix representing the complexified frame relative the above trivialisation, we can write

$$
\operatorname{Fr}\left(f_{i}\right)^{\uparrow}=F_{i} \cdot F .
$$

Here · denotes multiplication of $U(n)$-matrixes.

Recall that the spaces $V_{2 n, n+1}$ are $(n-2)$-connected and that

$$
\pi_{n-1}\left(V_{2 n, n+1}\right)=Y(n)= \begin{cases}\mathbf{Z} / 2, & n \text { even, } \\ \mathbf{Z}, & n \text { odd. }\end{cases}
$$

The Hurewicz isomorphism implies that, $H^{n-1}\left(V_{2 n, n+1} ; \mathbf{Z}\right) \cong Y(n)$. Fix a generator $g_{n} \in H^{n-1}\left(V_{2 n, n+1} ; \mathbf{Z}\right)$.

We start by showing

$$
\epsilon\left(f_{1}, f_{2}\right)=\left(p \circ F_{1}\right)^{*}\left(g_{n}\right)-\left(p \circ F_{1}\right)^{*}\left(g_{n}\right) .
$$

By Lemma 6.11, the two $S O(n)$-equivariant maps $s\left(f_{i}\right)$ are $S O(n)$-equivariantly homotopy if and only if the $S O(n-1)$-equivariant maps

$$
s\left(f_{i}\right)^{\uparrow}=p \circ\left(F_{i} \cdot F\right)=\rho_{F}\left(p \circ F_{i}\right)
$$

are $S O(n-1)$-equivariantly homotopic, where $\rho_{M}$ denotes the action on $V_{2 n, n+1}$ induced by multiplication on the right by a matrix $M$. This happens if and only if the maps $p \circ F_{i}$ are homotopic. The obstruction to the problem of finding a homotopy between the two maps

$$
p \circ F_{i}: L_{x} \rightarrow V_{2 n, n+1}
$$

is given by $\left(p \circ F_{1}\right)^{*}\left(g_{n}\right)-\left(p \circ F_{1}\right)^{*}\left(g_{n}\right)$, which implies the statement.

When $n$ is odd, $H^{n-1}\left(V_{2 n, n+1} ; \mathbf{Z}\right) \cong \mathbf{Z}$ and $H^{n-1}(U(n) ; \mathbf{Z})$ are both torsionfree, so we can tensor them by $\mathbf{Q}$ and get

$$
p^{*}: H^{n-1}\left(V_{2 n, n+1} ; \mathbf{Q}\right) \rightarrow H^{n-1}(U(n) ; \mathbf{Q})
$$

which factors through $H^{n-1}(S O(2 n) ; \mathbf{Q})$.

By looking at the spectral sequence (see Figure 6.4) of the fibration

$$
S O(n-1) \rightarrow S O(2 n) \rightarrow V_{2 n, n+1}
$$

we see that the pullback of $g_{n} \in H^{n-1}\left(V_{2 n, n+1}\right.$; Q $)$ to $H^{n-1}(S O(2 n)$; Q) vanishes: it lives in the $E_{n-1}^{0, n-1}$ space of the spectral sequence and is necessarily killed by the differential coming from

$$
E_{n-1}^{n-2,0}=H^{n-2}(S O(n-1) ; \mathbf{Q})
$$




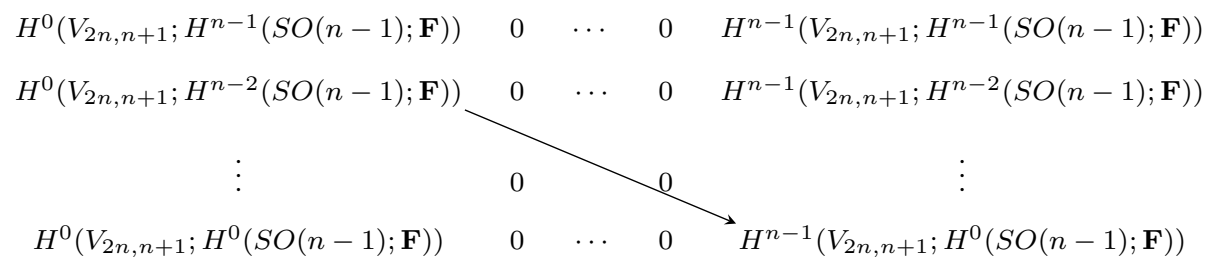

FIGURE 2. Part of the $E_{n-1}$-page of the spectral sequence for the fibration $S O(n-1) \rightarrow S O(2 n) \rightarrow V_{2 n, n+1}$. The coefficient field $\mathbf{F}$ is either $\mathbf{Q}$ if $n$ is odd or $\mathbf{Z} / 2$ if $n$ is even.

because

$$
\operatorname{rank} H^{n-2}(S O(2 n) ; \mathbf{Q})=\operatorname{rank} H^{n-2}(S O(n-1) ; \mathbf{Q})-1
$$

since $H^{n-2}(S O(n-1) ; \mathbf{Q})$ additionally contains the suspension of the Euler class. As a consequence, $p^{*} F_{i}^{*}\left(g_{n}\right)=0$ when $n$ is odd and hence

$$
\epsilon\left(f_{1}, f_{2}\right)=0 \text {. }
$$

When $n$ is even, we will show that $g_{n}$ pulls back to the suspension of $w_{n}$ in $H^{n-1}(S O(2 n) ; \mathbf{Z} / 2)$. To see this, consider the spectral sequence of the fibration $S O(n-1) \rightarrow S O(2 n) \rightarrow V_{2 n, n+1}$ for cohomology with coefficients in $\mathbf{Z} / 2$. We get

$$
H^{n-1}(S O(2 n) ; \mathbf{Z} / 2)=E_{\infty}^{n-1,0} \oplus E_{\infty}^{0, n-1}
$$

and since $H^{n-1}(S O(2 n) ; \mathbf{Z} / 2)$ has the same rank as

$$
E_{n-1}^{n-1,0} \oplus E_{n-1}^{0, n-1}=H^{n-1}(S O(n-1) ; \mathbf{Z} / 2) \oplus H^{n-1}\left(V_{2 n, n+1} ; \mathbf{Z} / 2\right)
$$

we know that the differential $E_{n-1}^{n-2,0} \rightarrow E_{n-1}^{0, n-1}$ vanishes and the pullback of $g_{n}$ to $H^{n-1}(S O(2 n) ; \mathbf{Z} / 2)$ survives. After pulling it back further to the fibre $H^{n-1}(S O(n-1) ; \mathbf{Z} / 2)$ it vanishes. This implies that the pullback of $g_{n}$ to $S O(2 n)$ must be the unique nonzero element in the kernel of the projection

$$
H^{n-1}(S O(2 n) ; \mathbf{Z} / 2) \rightarrow H^{n-1}(S O(n-1) ; \mathbf{Z} / 2),
$$

which is precisely the suspension of $w_{n}$. Pulling back further to $U(n)$ gives the suspension of the $\frac{n}{2}$ th Chern class reduced modulo 2; therefore

$$
\epsilon\left(f_{1}, f_{2}\right)=\Delta\left(f_{1}, f_{2}\right) \bmod 2 .
$$

Finally we will prove the statement about frame maps which agree on the $(n-2)$-skeleton. If the Lagrangian frame maps are homotopic when restricted to the $(n-2)$-skeleton of $L$, it follows that the same is true for the $S O(n-1)$-equivariant Lagrangian frame maps $\operatorname{Fr}\left(f_{i}\right)^{\uparrow}$. It follows that the value of

$$
\left(p \circ F_{1}\right)^{*}\left(g_{n}\right)-\left(p \circ F_{1}\right)^{*}\left(g_{n}\right)
$$


on an $(n-1)$-cell of $L$ can be obtained by evaluating $g_{n}$ on a spherical class, which moreover factorises as

$$
S^{n-1} \rightarrow U(n) \rightarrow V_{2 n, n+1} .
$$

Lemma 6.12 shows that $\epsilon\left(f_{1}, f_{2}\right)$ vanishes when $n \geq 5$.

6.5. Proof of Corollary C, To prove Corollary C, recall that the minimal Maslov number of a monotone torus is two (see the proof of Corollary 6.2), hence the Maslov class is primitive. The diffeomorphism group of the torus acts transitively on primitive first cohomology classes and hence by reparametrising we can assume that the Maslov classes of two monotone Lagrangian embeddings $f_{1}, f_{2}$ agree.

Now applying Lemma 6.8 and Proposition 6.10 we see that the HaefligerHirsch obstruction to smooth isotopy vanishes when $n$ is odd and at least five.

\section{Generalisations}

Recall that by Corollary 3.3 our unknottedness result Theorem 6.1 is already known for $S^{1} \times S^{3}$ and $S^{1} \times S^{7}$. It would be nice to recover this result using our techniques and to extend it to other products $S^{1} \times S^{n-1}$. Unfortunately we do not know how to do this in general. In this section we prove unknottedness in sufficiently high dimensions (starting with $S^{1} \times S^{4}$ ) with either a restriction on the minimal Maslov number or the condition of monotonicity.

Theorem 7.1. Let $n \geq 5$. Then the smooth knot type of a Lagrangian embedding $S^{1} \times S^{n-1} \rightarrow \mathbf{C}^{n}$ is determined by the $S O(n)$-equivariant homotopy type of the Lagrangian frame map restricted to the complement $L_{x}$ of a point $x \in L$ in the two cases:

- $n$ is even and the minimal Maslov number is $n$,

- $n$ is odd and the Lagrangian embedding is monotone.

Remark 7.2. Note that no monotonicity assumption is needed when the minimal Maslov number is $n>4$ because such Lagrangian embeddings are automatically monotone. This is because the first cohomology has rank one and because there exists a relative homology class of discs with strictly positive area and Maslov number between $3-n$ and $n+1$ [5, Theorem 2.1 and subsequent Remark 5]. Since this disc is essential in relative homology its Maslov number is a nonzero multiple of $n$ by the assumption on minimal Maslov number. When $n>4$, the only nonzero multiple of $n$ between $3-n$ and $n+1$ is $n$. When $n$ is even, examples of Lagrangian embeddings satisfying the hypotheses of the theorem are well-known 
and can be constructed by a suitable Polterovich surgery on the standard (Whitney) immersed exact Lagrangian sphere [38].

When $n$ is odd the odd-dimensional cohomology of the universal cover of $S^{1} \times S^{n-1}$ vanishes and hence (using monotonicity) Damian's theorem implies that the Lagrangian has minimal Maslov number 2. Moreover his theorem gives, as usual, a moduli space of holomorphic Maslov 2 discs with boundary on the Lagrangian such that the evaluation map $\mathcal{M}_{0,1}(L, \beta) \rightarrow L$ has degree one. Examples of monotone Lagrangians satisfying the hypotheses of the theorem when $n$ is odd can be constructed either by Polterovich surgery on the Whitney sphere as above, or by applying the construction of Audin-Lalonde-Polterovich [4] to exact Maslov zero Lagrangian immersions of even dimensional spheres (which exist by the h-principle for exact Lagrangian immersions).

The strategy of proof is the same as for Theorem 6.1

$n=2 k+2$ even. As in Lemma 6.7 we use the projection $\sigma: S^{1} \times S^{n-1} \rightarrow$ $S^{1}$ to find a nearby Lagrangian embedding $S^{1} \times S^{n-1}=L^{\prime} \rightarrow \mathbf{C}^{n}$ (the graph of $\epsilon d \sigma$ ) with minimal Maslov number $n$ and smaller monotonicity constant. We will pick a suitable almost complex structure $J$ and consider a suitable moduli space (see Lemma 7.3) $M(\gamma, J)$ of $J$-discs with boundary on $L^{\prime}$ representing the relative class $\beta \in H_{2}\left(\mathbf{C}^{n} \backslash L^{\prime} ; \mathbf{Z}\right) \cong H_{1}\left(L^{\prime} ; \mathbf{Z}\right)$ with Maslov number $2 k+2$. We will see that

- this moduli space admits a degree one evaluation map to $L^{\prime}$,

- by choosing $J$ suitably we can assume that all the discs in this moduli space avoid $L$.

This will imply that $\nabla \sigma$ is the Haefliger-Hirsch field so that Proposition 6.10 will then apply, proving Theorem 7.1. We begin by specifying the moduli space $M(\gamma, J)$.

Lemma 7.3. Let $\gamma: S^{1} \rightarrow L^{\prime}$ be a generic embedded loop representing the class $\beta \in H_{1}\left(L^{\prime} ; \mathbf{Z}\right)$ and $J$ a generic almost complex structure. Let $\mathcal{M}_{0,2}(\beta, J)$ denote the moduli space of $J$-discs with boundary on $L^{\prime}$ representing the class $\beta$ and two boundary marked points. Write $\mathrm{ev}_{1}, \mathrm{ev}_{2}: \mathcal{M}_{0,2}(\beta, J) \rightarrow L^{\prime}$ for the evaluation maps at the two marked points. Let $M(\gamma, J)=\operatorname{ev}_{2}^{-1}\left(\gamma\left(S^{1}\right)\right)$. Then the evaluation map

has degree \pm 1 .

$$
\left.\mathrm{ev}_{1}\right|_{M(\gamma, J)}: M(\gamma, J) \rightarrow L^{\prime}
$$

Proof. This is just a geometric interpretation of one of the $E_{1}$-differentials in the Biran-Cornea spectral sequence: the solid arrow in Figure 7 . Since the spectral sequence must collapse at the $E_{2}$ stage this differential must be an isomorphism over $\mathbf{Z}$. The differential is multiplication by $t$ times the 


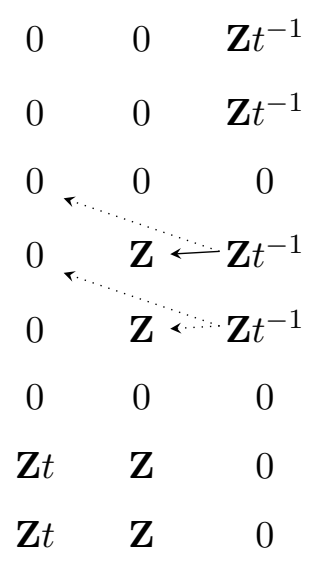

FIGURE 3. The $E_{1}$-page of the Biran-Cornea spectral sequence for a monotone Maslov 4 Lagrangian $S^{1} \times S^{3}$ in $\mathbf{C}^{2}$ with $E_{1}$ (horizontal) and $E_{2}$ (knight's move) differentials indicated.

degree of the evaluation map we are interested in. Therefore this degree is \pm 1 .

The $n=2 k+2$ case of Theorem 7.1 will now follow from the following result.

Proposition 7.4. There exists a loop $\gamma$ and a regular almost complex structure $J$ (obtained by neck-stretching) such that the discs in $M(\gamma, J)$ avoid $L$.

To prepare for the proof of this result, we state a version Lazzarini's decomposition theorem for punctured holomorphic discs with boundary on a Lagrangian.

Lemma 7.5 (Compare with [31, 32]). Let $X$ be a symplectic manifold with strong convex or concave contact-type boundary, let $\bar{X}$ denote its completion and $L \subset X$ be a compact Lagrangian submanifold. Let $J$ be a compatible almost complex structure on $\bar{X}$ adapted to the contact structure on the ends. Suppose that $u: \Sigma \rightarrow \bar{X}$ is a finite-energy punctured holomorphic disc with boundary on $L$. There exists a somewhere-injective finite-energy punctured disc $v: \Sigma^{\prime} \rightarrow \bar{X}$ with boundary on $L$ such that $v\left(\Sigma^{\prime}\right) \subset u(\Sigma)$.

Proof. Lazzarini's proof [32] carries over almost word-for-word. One must be careful to understand non-injectivity near the punctures, but this is controlled by Corollaries 2.5 and 2.6 of [41] which imply that, in a sufficiently small annular neighbourhood of a puncture, $u$ does indeed factor through 
a finite-energy embedded half-cylinder and that two half-cylinders asymptotic to the same puncture are either asymptotically disjoint or, asymptotically, their images coincide.

Proof of Proposition 7.4 Assume that Proposition 7.4 is false and that for all $\gamma$ and $J$ some disc in $M(\gamma, J)$ intersects $L$.

Fix the loop $\gamma$ in $L^{\prime}$ and let $J_{t}$ be a neck-stretching sequence of almost complex structures for $L$. Choose a Weinstein neighbourhood of $L$ and let $V$ denote its complement. We will later choose $I=\left.J_{t}\right|_{V}$ appropriately to derive a contradiction. By Lemma 7.3 , for all $t$ there is a $J_{t}$-holomorphic disc in $M\left(\gamma, J_{t}\right)$, that is a disc with boundary on $L^{\prime}$ representing the minimal area class $\beta$ and (by assumption) passing through $L$.

We can extract a Gromov-Hofer convergent subsequence of these discs whose limit is a holomorphic building $u=u_{\bar{W}} \cup u_{S_{1}} \cup \cdots \cup u_{S_{\ell}} \cup u_{\bar{V}}$ where $u_{\bar{W}}$ denotes the component in $\bar{W}=T^{*} L$ (the symplectic completion of the Weinstein neighbourhood), $u_{\bar{V}}$ denotes the component in $\bar{V}=\mathbf{C}^{n} \backslash L$ and $u_{S_{k}}$ denote components in the intermediate symplectisation levels. Let $v$ be the component of $u_{\bar{V}}$ with boundary on $L^{\prime}$. Note that the boundary of $v$ passes through the loop $\gamma$ by construction.

Claim 1. There are no other components in $u_{\bar{V}}$.

Proof. Another component would compactify to give a topological surface with boundary on $L$, and would represent a relative homology class in $H_{2}\left(\mathbf{C}^{n}, L ; \mathbf{Z}\right)$ with nonzero area since it is holomorphic. Since the monotonicity constant of $L$ is strictly bigger than that of $L^{\prime}$ the area of this component is strictly bigger than the area of $\beta$, which contradicts the conservation of area under Gromov-Hofer convergence [15, Definition 2.7(e), Theorem 2.9].

Claim 2. The curve $v$ has only contractible geodesics as asymptotes.

Proof. Assume that $v$ has a noncontractible geodesic as asymptote. Since the building as a whole has genus zero this asymptote must be the positive asymptote of another genus zero building in $\bar{W} \cup S_{1} \cup \cdots \cup S_{\ell^{\prime}} \cup \bar{V}$ which glues topologically to give a plane in $\mathbf{C}^{n}$. Since the geodesic is noncontractible this building cannot lie entirely in $\bar{W} \cup S_{1} \cup \cdots \cup S_{\ell^{\prime}}$ or else it would glue topologically to give a nullhomotopy of the geodesic. Therefore there must be another component of $\bar{V}$, contradicting Claim 1

Claim 3. The curve $v$ is somewhere injective. 
Proof. By Lemma 7.5 we can extract a somewhere injective punctured disc $w^{\prime}$ whose image is a subset of $w$. Suppose that $w^{\prime}$ is not just a reparametrisation of $w$. Since the asymptotes are contractible geodesics in $L$ we can cap them off with discs in $L$ with no symplectic area and obtain a topological disc in $\mathbf{C}^{n}$ with boundary on $L$ whose area is strictly smaller than that of $\beta$, which contradicts minimality of $\beta$.

Note that Claim 3 applies to all finite-energy punctured discs in the same moduli space as $v$ : we only use the fact that the asymptotes are contractible geodesics and that the result of topologically capping these asymptotes is homologous to $\beta$.

In the standard way [17], we can achieve transversality for moduli spaces of somewhere-injective finite-energy punctured discs by perturbing $I=\left.J_{t}\right|_{V}$. In particular the moduli space $S$ of punctured discs containing $v$ is smooth and of the expected dimension. Similarly, if we equip punctured discs with a boundary marked point, we can assume that the resulting evaluation map ev : $S \rightarrow L^{\prime}$ is transverse to $\gamma$.

Claim 4. When $n>4$ the expected dimension of $\mathrm{ev}^{-1}(\gamma) \subset S$ is negative. In particular, when I is chosen generically this set is empty.

Proof. Using Equation (9.3) from Section 9, the expected dimension formula for punctured discs in $V$ with $s_{-}$negative punctures asymptotic to Reeb orbits, the $i$ th of which covers a contractible geodesic in $S^{n-1}$ with multiplicity $m_{i}$, is

$$
(n-3)\left(1-s_{-}\right)+\mu-\sum_{i=1}^{s_{-}}\left(2 m_{i}-1\right)(n-2)
$$

Since $\mu=n$, the expected dimension is:

$$
2 n-3-\sum_{i=1}^{s_{-}}\left(n-3+\left(2 m_{i}-1\right)(n-2)\right)
$$

If we add a marked point on the boundary and require this to pass through the codimension $n-1$ loop $\gamma$ then the expected dimension becomes

$$
2 n-3-\sum_{i=1}^{s_{-}}\left(n-3+\left(2 m_{i}-1\right)(n-2)\right)+1-(n-1) \leq 4-n
$$

with equality if and only if $s_{-}=1$ and $m_{1}=1$. When $n>4$ this implies the claim.

Since $v$ is supposed to belong to this empty moduli space we get a contradiction. This completes the proof of Proposition 7.4 and therefore the proof of Theorem 7.1 in the case $n=2 k+2$. 
$n=2 k+1$ odd. In this case Damian's theorem from [16] implies that, for a regular $J$, the evaluation map

$$
\text { ev : } \mathcal{M}_{0,1}(\beta, J) \rightarrow L
$$

(from the moduli space of $J$-discs with boundary on $L$ representing the class $\beta=\left[S^{1}\right] \times\{\star\} \in H_{1}(L ; \mathbf{Z})$ and having one boundary marked point) has nonzero degree (in fact degree \pm 1 ). We argue in the usual way to prove that the vector field $\partial_{\theta}\left(\theta\right.$ being the coordinate on $\left.S^{1}\right)$ is a Haefliger-Hirsch field: push $L$ off along $J \partial_{\theta}$ to obtain a Lagrangian $L^{\prime}$ with smaller monotonicity constant and study the moduli space of discs with boundary on $L^{\prime}$ (for which the corresponding evaluation map still has nonzero degree). Assuming that for every $J$ there is a $J$-disc on $L^{\prime}$ in the class $\beta$ which intersects $L$ we use a neck-stretching sequence $J_{t}$ (stretching around $L$ ) and extract a Gromov-Hausdorff convergent subsequence of $J_{t}$-discs which intersect $L$. One component is a plane and it is possible that this plane lives in the Weinstein neighbourhood of $L$. As in the case $n=2 k+2$ we will argue that this cannot occur for generic neck-stretching sequences provided that $n>4$. Claims 1-3 still apply and we just need to understand what replaces Claim 4.

By Claims 1-3, the part $u_{\bar{V}}$ of the limit building living in the complement of $L$ consists of a single punctured disc with boundary on $L^{\prime}$ and having contractible geodesics as asymptotes. The expected dimension for moduli spaces of such discs is

$$
(n-3)\left(1-s_{-}\right)+\mu-\sum_{i=1}^{s_{-}}\left(2 m_{i}-1\right)(n-2)
$$

where now $\mu=2$ and $m_{i}$ is the number of times the $i$ th Reeb orbit wraps around the underlying simple geodesic. The expected dimension is therefore

$$
n-1-\sum_{i=1}^{s_{-}}\left(n-3+\left(2 m_{i}-1\right)(n-2)\right)
$$

which is at most $n-1-(n-3+n-2)=4-n$ in the worst case $s_{-}=1$, $m_{1}=1$. If $n>4$ this is negative so this kind of breaking is generically prohibited.

This completes the proof of Theorem[7.1 in the case $n=2 k+1$.

7.1. Proof of Corollary E, Let $f_{1}, f_{2}: L=S^{1} \times S^{n-1} \rightarrow \mathbf{C}^{n}$ be two Lagrangian embeddings which both satisfy one of the assumptions in Theorem 7.1. Let $\sigma$ be the projection onto the $S^{1}$-factor. Since $n>2$, the standard cell decomposition of $L$ has $(n-2)$-skeleton $S^{1} \times\{p t\}$. If $n$ is even then the Maslov class is $\pm n[d \sigma]$ by assumption. If $n$ is odd then by monotonicity and Damian's theorem [16] we know that the Maslov class is $\pm 2[d \sigma]$. In either case, by reparametrising with a reflection of $S^{1}$ if necessary, we can 
assume that the Maslov class of $f_{1}$ and the Maslov class of $f_{2}$ agree, which ensures that the Lagrangian frame maps are homotopic when restricted to the $(n-2)$-skeleton of $L$.

By Theorem 7.1. Proposition 6.10 applies, in particular the last statement showing that the difference class $\epsilon\left(f_{1}, f_{2}\right)$ vanishes. This finishes the proof of Corollary E,

\section{CONSTRUCTING KNOTTED LAGRANGIAN TORI}

When $n$ is even we will construct smoothly non-isotopic monotone Lagrangian tori. Let $m: T^{n-1} \rightarrow U(n-1)$ be a map such that the suspension of the Chern class $c_{n / 2}$ is nontrivial and not divisible by two. For instance the inclusion of a maximal torus would suffice. Using the h-principle for exact Lagrangian immersions, let $g_{0}$ and $g_{1}$ be exact Lagrangian immersions whose Lagrangian frame maps are, respectively, nullhomotopic and homotopic to $m$. Apply the Audin-Lalonde-Polterovich construction to obtain two embedded Lagrangians diffeomorphic to $S^{1} \times T^{n-1}$ in $\mathbf{C}^{n}$ which are monotone by exactness of the immersions and by the way we have chosen their Gauss maps. By construction the difference class is nonzero. Analogous examples of smoothly knotted $S^{1} \times S^{3}$ s with non-homotopic Gauss maps were constructed by Borrelli [8].

In particular, consider the Clifford (product) torus. If we use the trivialisation of $T T^{n}$ coming from its structure as a Lie group then we get a trivialisation of $T_{\mathbf{C}} T^{n}$ and with respect to this trivialisation the Lagrangian frame map $T^{n} \rightarrow U(n)$ of the Clifford torus is just just $i$ times the inclusion of a maximal torus. If we instead apply the Audin-Lalonde-Polterovich construction to an immersed exact Lagrangian $T^{n-1} \rightarrow \mathbf{C}^{n-1}$ with nullhomotopic frame map $T^{n-1} \rightarrow U(n-1)$ (relative to the same trivialisation) then we get a monotone Lagrangian torus which is not smoothly isotopic to the Clifford torus, provided $n$ is even.

\section{APPENDIX: INDEX FORMULA FOR PSEUDOHOLOMORPHIC CURVES}

Consider the symplectic manifold $V=\mathbf{C}^{n} \backslash L$ with a negative cylindrical end. In this section we will describe the Fredholm index of the linearised $\bar{\partial}$-operator for pseudoholomorphic discs in $V$ having boundary on a Lagrangian submanifold $L^{\prime} \subset V$ and internal boundary punctures asymptotic to Reeb orbits of the negative cylindrical end. Since we are interested in the case when the negative end corresponds to $(-\infty, 0] \times S_{\rho, g}^{*} L$, where the metric $g$ is non-degenerate in the Bott sense, we also consider index formulas for pseudoholomorphic curves having punctures asymptotic to Reeb orbits which are non-degenerate in the Bott sense. 
9.1. The generalised Conley-Zehnder index. We start by a brief description of the Conley-Zehnder indices of the Reeb orbits in this situation. The Conley-Zehnder index $\mu_{C Z}(\gamma)$ of a Reeb orbit $\gamma$ inside a contact manifold $(Y, \xi=\operatorname{ker} \lambda)$ can be computed as follows, following [40, Remark 5.4]. First, we fix a symplectic trivialisation of the contact distribution $\xi$ along $\gamma$, in which the linearised Reeb flow is expressed as a path of symplectic matrices $\Psi_{t}$. One gets an induced path of Lagrangian planes inside $\left(\mathbf{C}^{n} \oplus \mathbf{C}^{n},\left(-\omega_{0}\right) \oplus \omega_{0}\right)$ parametrised by

$$
\left(\mathrm{Id}, \Psi_{t}\right): \mathbf{C}^{n} \rightarrow \mathbf{C}^{n} \oplus \mathbf{C}^{n}
$$

for which one can compute the Maslov index as defined in [40, Section 2] with respect to the Lagrangian reference plane consisting of the diagonal. This is the Conley-Zehnder index.

Observe that this index is defined even in the case when 1 is an eigenvalue of the return-map of the linearisation, but that it can take half-integer values in this case. In the case when there is a Bott manifold $S$ of Reeb orbits, we will use $\mu_{C Z}(S)$ to denote the Conley-Zehnder index of a Reeb orbit in this family.

\subsection{The Fredholm index for a pseudoholomorphic curve with punctures} in the Bott case. In [10, Corollary 5.4] the formula for the Fredholm index of the linearised $\bar{\partial}$-operator for a closed pseudoholomorphic curve inside a symplectic $2 n$-dimensional manifold $X$ with cylindrical ends is generalised to the case where the Reeb orbits are nondegenerate in the Bott sense. It is shown that a closed pseudoholomorphic curve $C$ of genus $g$ having internal punctures asymptotic to Reeb orbits in the families $S_{1}^{+}, \ldots, S_{s^{+}}^{+}$and $S_{1}^{-}, \ldots, S_{s^{-}}^{-}$at positive and negative ends, respectively, the Fredholm index satisfies

$$
\begin{aligned}
\operatorname{index}(C)= & (n-3)\left(2-2 g-s^{+}-s^{-}\right)+2 c_{1}^{\mathrm{rel}}(C)+ \\
& +\sum_{i=1}^{s^{+}}\left(\mu_{C Z}\left(S_{i}^{+}\right)+\frac{1}{2} \operatorname{dim} S_{i}^{+}\right)-\sum_{i=1}^{s^{-}}\left(\mu_{C Z}\left(S_{i}^{-}\right)-\frac{1}{2} \operatorname{dim} S_{i}^{-}\right) .
\end{aligned}
$$

Here $c_{1}^{\mathrm{rel}}(C)$ denotes the first Chern number of the bundle $T X$ pulled back to $C$ and extended over the punctures using the trivialisation of $\left.T X\right|_{\gamma}=$ $\left.\mathbf{C} \oplus \xi\right|_{\gamma}$ chosen above along the Reeb orbits.

In the case when the curve $C$ has boundary $\partial C$ on a Lagrangian submanifold $L^{\prime} \subset X$, given any trivialisation of $T X$ along the boundary, there is an induced Maslov index of $L^{\prime}$ along this boundary which we denote by 
$\mu(\partial C)$. One can deduce that

$\operatorname{index}(C)=(n-3)\left(1-2 g-s^{+}-s^{-}\right)+\mu(\partial C)+2 c_{1}^{\text {rel }}(C)+$

$$
+\sum_{i=1}^{s^{+}}\left(\mu_{C Z}\left(S_{i}^{+}\right)+\frac{1}{2} \operatorname{dim} S_{i}^{+}\right)-\sum_{i=1}^{s^{-}}\left(\mu_{C Z}\left(S_{i}^{-}\right)-\frac{1}{2} \operatorname{dim} S_{i}^{-}\right) .
$$

Here $c_{1}^{\mathrm{rel}}(C)$ denotes the first Chern number of $T X$ pulled back to $C / \partial D$ using the trivialisation of $\left.T X\right|_{\partial D}$ chosen above and extended to the punctures as before.

In the case when the first Chern class $c_{1}$ vanishes for $X$, the above ConleyZehnder index is canonically defined for nullhomologous Reeb orbits in any trivialisation induced by a choice of bounding chain. Likewise, the Maslov index is canonically defined for any path on $L^{\prime}$ which is nullhomologous in $X$. This follows from the fact that two different choices of bounding chains $A$ and $B$ will give rise to a difference of $2 c_{1}(A-B)$ in the respective index. Finally, in the case $c_{1}=0$, it also follows that the term $2 c_{1}^{\mathrm{rel}}(C)$ vanishes for these choices of trivialisations.

9.3. The index formula for discs in $V$ with internal punctures. Consider the symplectic manifold $V=\mathbf{C}^{n} \backslash L$, where $L \cong S^{1} \times S^{n-1}$ is a Lagrangian submanifold. We view $V$ as a symplectic manifold with a negative end corresponding to $(-\infty, 0] \times S_{\rho, g}^{*}\left(S^{1} \times S^{n-1}\right)$, where $g$ is the product metric on $S^{1} \times S^{n-1}$ for the round metric on $S^{n-1}$. We are now ready to show the following result for discs inside $V$ having boundary on a Lagrangian submanifold $L^{\prime} \subset V$.

For the Maslov index of a closed curve on $L^{\prime} \subset V$ we use the trivialisation of $T V \subset T \mathbf{C}^{n}$ induced by the canonical trivialisation of $T \mathbf{C}^{n}$. Observe that any loop on $L^{\prime}$ is contractible inside $V$ whenever $n>2$ and that the induced trivialisation by any chain bounding a loop on $L^{\prime}$ agrees with this trivialisation.

Lemma 9.2. A pseudoholomorphic disc $D$ with boundary on $L^{\prime} \subset V$ having a number $s^{-}$of internal punctures asymptotic to the families $S_{1}^{-}, \ldots, S_{s^{-}}^{-}$of Reeb orbits at the negative end of $V$, where $S_{i}$ corresponds to a family of $m_{i}$-multiple covers of closed contractible geodesics on $S^{1} \times S^{n-1}$, has Fredholm index

$$
\operatorname{index}(D)=(n-3)\left(1-s^{+}\right)+\mu(\partial C)-\sum_{i=1}^{s^{+}}\left(2 m_{i}-1\right)(n-2) .
$$

Proof. For the trivialisation of the contact distribution $\left.\xi\right|_{\gamma}$ along a Reeb orbit $\gamma$ of $S_{\rho, g}^{*} L$ we choose the trivialisation which is induced by a trivialisation of the vertical subbundle of $\xi$, which is a Lagrangian subspace. Observe that this trivialisation agrees for the canonical trivialisation of $\xi$ along any 
Reeb orbit on $S_{\rho, g}^{*} L$ which is nullhomologous. Moreover, since $c_{1}$ vanishes for $V$, this means that the term $c_{1}^{\text {rel }}$ vanishes in the index formula.

\section{Consider the energy functional}

$$
E(\eta)=\int_{S^{1}}\|\eta(\theta)\|^{2} d \theta
$$

for closed curves $\eta$ in $L$. Let $H$ denote the Hessian of $E$ at a critical point $\gamma$, which corresponds to a closed geodesic on $L$ parametrised by path of constant speed. We let $\iota(\gamma)$ and $\nu(\gamma)$ denote the dimension of negativedefinite eigenspace and nullity of $H$ at $\gamma$, respectively.

We let $\gamma$ be a geodesic on $S^{1} \times S^{n-1}$ and $\widetilde{\gamma}$ be Reeb orbit in $S_{\rho, g}^{*}\left(S^{1} \times S^{n-1}\right)$ corresponding to the cogeodesic lift. The lemma follows by combining Lemma 9.4 below together with Equation (9.1) above, and the formula

$$
\mu_{C Z}(\widetilde{\gamma})=\iota(\gamma)+\frac{1}{2} \nu(\gamma)
$$

proved in [14, Equation 60], which holds in the canonical trivialisation.

Lemma 9.4. Let $S^{1} \times S^{n-1}$ be endowed with the product metric, where the metric on the factor $S^{n-1}$ is the round metric. A closed contractible geodesic $\gamma$ on $S^{1} \times$ $S^{n-1}$, which moreover is the $m$-fold cover of a simply covered geodesic, has Morseindex and nullity satisfying

$$
\iota(\gamma)=(2 m-1)(n-2), \quad \nu(\gamma)=n .
$$

Proof. Since geodesics on $S^{1} \times S^{n-1}$ project to geodesics on either factor, it follows that any geodesic which starts and ends on the hypersurface $\{t\} \times S^{n-1}$ must either be a geodesic contained entirely in this hypersurface, or must wrap around the $S^{1}$-direction a nonzero number of times. Furthermore, a geodesic contained in this hypersurface is a geodesic on the round $S^{n-1}$.

From this it follows that a contractible geodesic on $S^{1} \times S^{n-1}$ is contained in such a hypersurface, and that broken Jacobi fields along such a geodesic correspond bijectively to broken Jacobi fields on the corresponding geodesic on the round $S^{n}$. In addition, the only non-broken Jacobi field along a closed geodesic in $\{t\} \times S^{n-1}$ which does not arise as a Jacobi field on $S^{n-1}$ is the Jacobi field induced by a rotation of the $S^{1}$-factor.

The formulae on [9. Page 346] for the index and nullity of a geodesic on $S^{n-1}$ now implies the result.

\section{ACKNOWLEDGEMENTS}

The authors would like to acknowledge helpful discussions and communications with Paul Biran, Felix Schlenk and Chris Wendl. The paper [8] 
proved extremely helpful in crystallising our proof. Our collaboration is supported by a travel grant from the Contact And Symplectic Topology network (CAST) which is funded by the European Science Foundation (ESF).

\section{REFERENCES}

[1] P. Albers. On the extrinsic topology of Lagrangian submanifolds. Int. Math. Res. Not., (38):2341-2371, 2005. 3.5

[2] P. Albers. Erratum for "On the extrinsic topology of Lagrangian submanifolds" [MR2180810]. Int. Math. Res. Not. IMRN, (7):1363-1369, 2010.3.5

[3] V. I. Arnol'd. On a characteristic class entering into conditions of quantization. Funkcional. Anal. i Priložen., 1:1-14, 1967.2

[4] M. Audin, F. Lalonde, and L. Polterovich. Chapter X. Symplectic rigidity: Lagrangian submanifolds. In M. Audin and J. Lafontaine, editors, Holomorphic curves in symplectic geometry, volume 117 of Progress in Mathematics, pages 271-321. Birkhäuser, 1994.77.2

[5] P. Biran and K. Cieliebak. Lagrangian embeddings into subcritical Stein manifolds. Israel J. Math., 127:221-244, 2002.7.2

[6] P. Biran and O. Cornea. A Lagrangian quantum homology. In New perspectives and challenges in symplectic field theory, volume 49 of CRM Proc. Lecture Notes, pages 1-44. Amer. Math. Soc., Providence, RI, 2009.2

[7] M. S. Borman, T.-J. Li, and W. Wu. Spherical Lagrangians via ball packings and symplectic cutting. arXiv:1211.5952, 2012.3.4

[8] V. Borrelli. New examples of Lagrangian rigidity. Israel J. Math., 125:221-235, 2001. 1. 1, 1, 1, 3.1, 3.2, 3.3, $8,9.3$

[9] R. Bott. Lectures on Morse theory, old and new. Bull. Amer. Math. Soc. (N.S.), 7(2):331358, 1982. 9.3

[10] F. Bourgeois. A Morse-Bott approach to contact homology. PhD thesis, Stanford University, 2002. 9.2

[11] F. Bourgeois, Ya. Eliashberg, H. Hofer, K. Wysocki, and E. Zehnder. Compactness results in symplectic field theory. Geom. Topol., 7:799-888, 2003. 4

[12] L. Buhovsky. The Maslov class of Lagrangian tori and quantum products in Floer cohomology. J. Topol. Anal., 2(1):57-75, 2010.5 6.1

[13] Yu. V. Chekanov and F. Schlenk. Notes on monotone Lagrangian twist tori. Electron. Res. Announc. Math. Sci., 17:104-121, 2010.1

[14] K. Cieliebak and U. A. Frauenfelder. A Floer homology for exact contact embeddings. Pacific J. Math., 239(2):251-316, 2009.9.3

[15] K. Cieliebak and K. Mohnke. Compactness for punctured holomorphic curves. J. Symplectic Geom., 3(4):589-654, 2005. Conference on Symplectic Topology. 4.4 . 7

[16] M. Damian. Floer homology on the universal cover, a proof of Audin's conjecture and other constraints on Lagrangian submanifolds. Commentarii Mathematici Helvetici, 87:433-463, 2012. 5, 6.1, 7, 7.1.

[17] D. L. Dragnev. Fredholm theory and transversality for noncompact pseudoholomorphic maps in symplectizations. Comm. Pure Appl. Math., 57(6):726-763, 2004.7

[18] Ya. Eliashberg and L. Polterovich. Unknottedness of Lagrangian surfaces in symplectic 4-manifolds. Internat. Math. Res. Notices, (11):295-301, 1993. 1], 3.2, 3.4

[19] Ya. Eliashberg and L. Polterovich. New applications of Luttinger's surgery. Comment. Math. Helv., 69(4):512-522, 1994. 1, 3.1, 3.2

[20] Ya. Eliashberg and L. Polterovich. Local Lagrangian 2-knots are trivial. Ann. of Math. (2), 144(1):61-76, 1996. 1, 3.3

[21] Ya. Eliashberg and L. Polterovich. The problem of Lagrangian knots in four-manifolds. In Geometric topology (Athens, GA, 1993), volume 2 of AMS/IP Stud. Adv. Math., pages 313-327. Amer. Math. Soc., Providence, RI, 1997.1 
[22] J. D. Evans. Lagrangian spheres in del Pezzo surfaces. J. Topol., 3(1):181-227, 2010. 1 . 3.4

[23] J. D. Evans and J. Kędra. Remarks on monotone Lagrangians in $\mathbf{C}^{n}$. arXiv:1110.0927, 2011.2.7, 2. 5

[24] K. Groh, M. Schwarz, K. Smoczyk, and K. Zehmisch. Mean curvature flow of monotone Lagrangian submanifolds. Math. Z., 257(2):295-327, 2007.2

[25] M. L. Gromov. A topological technique for the construction of solutions of differential equations and inequalities. In Actes du Congrès International des Mathématiciens (Nice, 1970), Tome 2, pages 221-225. Gauthier-Villars, Paris, 1971.2.2

[26] A. Haefliger and M. W. Hirsch. On the existence and classification of differentiable embeddings. Topology, 2:129-135, 1963. 1, 6.2, 6.5. 6.6

[27] R. Hind. Lagrangian spheres in $S^{2} \times S^{2}$. Geom. Funct. Anal., 14(2):303-318, 2004. 1 . 3.4

[28] R. Hind. Lagrangian unknottedness in Stein surfaces. Asian J. Math., 16(1):1-36, 2012. 3.4

[29] R. Hind and A. Ivrii. Isotopies of high genus Lagrangian surfaces. arXiv:math/0602475, 2006. 1, 3.2, 3.4

[30] M. A. Kervaire. Some nonstable homotopy groups of Lie groups. Illinois J. Math., 4:161$169,1960.6 .4$

[31] L. Lazzarini. Decomposition of a $J$-holomorphic curve. Preprint. Can be downloaded at http: //www.math.jussieu.fr/ lazzarin/articles.html. 4.5 .5

[32] L. Lazzarini. Existence of a somewhere injective pseudo-holomorphic disc. Geom. Funct. Anal., 10(4):829-862, 2000.7.5.7

[33] J. A. Lees. On the classification of Lagrange immersions. Duke Math. J., 43(2):217-224, 1976.2.2

[34] T.-J. Li and W. Wu. Lagrangian spheres, symplectic surfaces and the symplectic mapping class group. Geom. Topol., 16(2):1121-1169, 2012.3.4

[35] K. M. Luttinger. Lagrangian tori in $\mathbf{R}^{4}$. J. Differential Geom., 42(2):220-228, 1995. 1. 3.1. 3.1. 3.2

[36] L. A. Lyusternik and A. I. Fet. Variational problems on closed manifolds. Doklady Akad. Nauk SSSR (N.S.), 81:17-18, 1951.6.3

[37] Y.-G. Oh. Floer cohomology of Lagrangian intersections and pseudo-holomorphic disks. I. Comm. Pure Appl. Math., 46(7):949-993, 1993.2

[38] L. Polterovich. The surgery of Lagrange submanifolds. Geom. Funct. Anal., 1(2):198210, 1991.7.2

[39] V. V. Prasolov. Elements of homology theory, volume 81 of Graduate Studies in Mathematics. American Mathematical Society, Providence, RI, 2007. Translated from the 2005 Russian original by Olga Sipacheva. 6.2

[40] J. Robbin and D. Salamon. The Maslov index for paths. Topology, 32(4):827-844, 1993. 9.1

[41] R. Siefring. Relative asymptotic behavior of pseudoholomorphic half-cylinders. Comm. Pure Appl. Math., 61(12):1631-1684, 2008.7

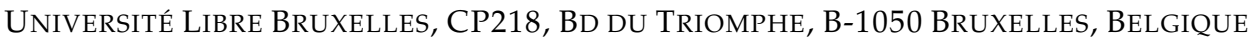

E-mail address: georgios.dimitroglou@ulb.ac.be

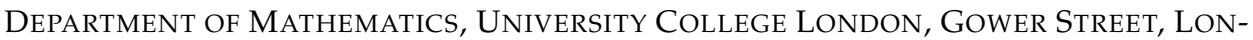
DON WC1E 6BT, UNITED KINGDOM

E-mail address: j.d.evans@ucl.ac.uk 